\title{
A Study of Heart Failure Patients and their Blood Pressure Trends Preceding Dementia Diagnosis
}

\author{
Wilson CK, Pangle AK, Wei JY, Azhar G* \\ Department of Geriatrics, Donald W. Reynolds Institute on Aging, UAMS, Little Rock, Arkansas, USA
}

\begin{abstract}
Introduction: Blood pressure and perfusion of the brain are central components of neurological health that are often influenced by Heart Failure. However, the connection between blood pressure and the development of dementia is not clearly understood.

Aim of the study: The aim of this retrospective study was to assess blood pressure trends in older heart failure patients and evaluate whether they developed dementia over the course of a five-year period.

Methods: This retrospective case-control study analyzed blood pressure changes preceding the diagnosis of dementia in patients over the age-of- 60 . Blood pressures were obtained from the date of dementia diagnosis, and then one-year and five years before diagnosis. Study "controls" were age-matched patients without dementia, using the mean-age-of dementia diagnosis as the first data point.

Results: $67.2 \%$ of patients with a dementia diagnosis had experienced a decrease in systolic pressure up to 5-years prior to their diagnosis, as compared to $46.6 \%$ of patients who had not been diagnosed by the same age. Similarly, in the one-year preceding the diagnosis, $62.7 \%$ of patients with dementia showed a decreasing systolic pressure over the time period, as compared to $50.0 \%$ of those who did not develop dementia. Additionally, there was a significant difference $(p<0.001)$ in the dementia rates between African American and Caucasian subgroups $(55.0 \%$ African Americans vs. $31.0 \%$ Caucasians).

Conclusions: Patients with dementia were more likely to have decreasing systolic pressure trends compared to age-matched patients without dementia and appeared to have significantly lower systolic pressures one-year-before dementia diagnosis. Lower systolic pressure can potentially reduce the perfusion pressure of the brain and cause subtle cerebral ischemia, leading to cognitive decline. It is very important that healthcare providers monitor these trends and risk factors as they manage blood pressures in geriatric patients.
\end{abstract}

Keywords: Blood pressure; Dementia; Gerontology; Race/ethnicity; Cardiovascular diseases and risk

\section{INTRODUCTION}

Dementia is a complex disease with multifactorial pathogenesis that carries a significant disease burden on society. It was estimated that in 2014, nearly 5 million of those 65-years and older had been diagnosed with dementia in the United States, an amount that is projected to nearly double by 2030 [1]. The complex myriad of risk factors that lead to the onset of dementia is not entirely understood but it is clear that cerebral perfusion and associated blood pressure plays a role in the health of the brain and the development of dementia [2]. This important relationship has been demonstrated at several points within the lifecycle of humans. For example, it has been shown that hypertension and prehypertension in midlife significantly increase the risk of dementia later in life $[3,4]$. Additionally, higher rates of dementia have been associated with both the persistence of elevated blood pressure in later life and a rapid decline in blood pressure during the same time period [4]. Further evidence has shown that even day-to-day variability of blood pressure in those greater than 60 years old may also i.e remove - confer greater risk of dementia [5]. It is likely that the influence blood pressure carries in cerebral health is mediated by changes in perfusion of cerebral tissue. It has been previously demonstrated that declining blood pressure may cause hypoperfusion of cerebral tissue, potentially leading to decreased gray matter volume and cerebral dysfunction $[2,6]$.

Correspondence to: Azhar G, M.D., Professor/Geriatrician, Department of Geriatrics, Donald W. Reynolds Institute on Aging, UAMS, Little Rock, Arkansas, USA, Tel: (501) 686-5884; E-mail: azhargohar@uams.edu

Received: January 24, 2020, Accepted: February 07, 2020, Published: February 14, 2020

Citation: Wilson CK, Pangle AK, Wei JY, Azhar G (2020) A Study of Heart Failure Patients and their Blood Pressure Trends Preceding Dementia Diagnosis, J Gerontol Geriatr Res 9:508. doi: 10.35248/2167-7182.20.9.508

Copyright: (C) 2020 Wilson CK, et al. This is an open-access article distributed under the terms of the Creative Commons Attribution License, which permits unrestricted use, distribution, and reproduction in any medium, provided the original author and source are credited. 
Heart failure is understood as a condition in which the heart is unable to adequately perfuse the body at normal physiologic pressures. Data from 2018 show that there were approximately 1 million new cases of heart failure in the U.S. with increasing incidence associated with increasing age [7]. In several regions of Europe, the prevalence of hypertension in heart failure patients has been estimated between $43-72 \%$ [8]. In opposition to the wellestablished linear relationship between elevated blood pressure and the risk of cardiovascular events in the general population, analysis of heart failure patients has demonstrated a J-curve for all-cause mortality, with both upper and lower ends of the blood pressure curve leading to higher mortality $[8,9]$. This leads to the postulation that in this unique population low systolic blood pressure may be harmful or, alternatively, a marker of severe disease. Therefore, those with heart failure may be at greater risk of the effects of hypoperfusion on cognitive health compared to individuals without heart failure.

It has been previously demonstrated that heart failure patients have a greater rate of all-cause dementia compared to the general population [10-13]. One Danish nationwide study demonstrated that patients with heart failure had about a 20\% greater risk of all-cause dementia compared to the general population [10]. An additional study has shown that late-life heart disease, including heart failure, were associated with higher risk of dementia and Alzheimer's disease [11]. In addition, the presence of heart failure and low diastolic blood pressure has been shown to additively increase the risk of dementia and Alzheimer's disease [12]. Finally, low cardiac index has been linked to increased rates of dementia with increasing risk for each subsequent standard deviation below the mean [13].

While these studies demonstrate a clear connection between heart failure and dementia, the mechanism is not entirely established. In the present study, we sought to clarify whether sudden changes in blood pressure in heart failure patients may be linked to rates of dementia diagnoses. Our overarching hypothesis was that a reduction in blood pressure below a person's baseline can cause neuronal damage and lead to long term cognitive decline. We therefore performed a retrospective analysis of data to look at trends in blood pressures, investigating whether there would be a greater reduction in blood pressure in the years preceding a diagnosis of dementia compared to age-matched controls that did not develop dementia.

This study is important because the effect of a decrease in blood pressure on the brain is not as well-appreciated as that of an increase in blood pressure. The emphasis in the past has always been to reduce blood pressure without considering the possibility that in some instances it could also lower perfusion pressure of the brain. This is particularly important in older individuals who are already pre-disposed to developing cognitive impairment or different types of dementia. Since we wanted to study changes in blood pressure before the diagnosis of dementia, we were limited to a retrospective design even with its limitations of missing data and many variables. Nevertheless, we did have significant results from this study and demonstrated clearly that in patients with dementia, there was a significant reduction in systolic blood pressure at five years and at one-year preceding diagnosis. This is clinically relevant information, supporting the need for healthcare providers, patients, and caregivers to monitor changes in blood pressure and pay attention to the connection between low blood pressure, cerebral perfusion and changes in cognition.

\section{RESEARCH METHODOLOGY}

This was a retrospective study wherein the University of Arkansas for Medical Sciences (UAMS) Data Warehouse was commissioned in 2017 to extract the records of heart failure patients seen at a geriatric outpatient clinic in Little Rock, Arkansas from 2011 to 2016. The study was approved by the local Institutional Review Board (Protocol\#134775). The UAMS Data Warehouse request generated a list of 281 patients over the age-of-60; we then retrospectively assessed the Electronic Medical Records (EMR) of these patients, 109 of whom had a diagnosis of dementia documented in their record. The mean age of the patients at the time of dementia diagnosis was $81 \pm 7.3$ yrs. All diagnoses of dementia were included in the study and no sub-types were excluded. No patients were contacted during the course of the study and all data were extracted directly from the records. Patient demographics are displayed in Table 1.

We aimed to evaluate decreasing blood pressure trends in the years preceding a dementia diagnosis in patients with heart failure to help provide more insight into the influence that heart failure and cerebral perfusion may have in the development of dementia. Our primary goal was to examine if there were a reduction in systolic or diastolic pressures at some point before dementia was diagnosed. Our secondary goal was to investigate whether the decrease in blood pressures was significantly different between patients who later developed dementia or patients of the same age range who did not develop dementia.

For patients with a diagnosis of dementia, systolic and diastolic blood pressure values were extracted from the EMR on the date dementia was added to the patient problem list $\left(\mathrm{T}_{0}\right)$, one-year prior to diagnosis (T-1), and five years prior to diagnosis (T-5). If blood pressure values for the date of dementia diagnosis were not available, the patient was not included in analysis. Patients who did not have a diagnosis of dementia were age-matched using the mean age at diagnosis for dementia patients. This mean age was used to delineate the first blood pressure data point $\left(\mathrm{T}_{0}\right)$ in the patients without dementia, while additional blood pressures for this group were obtained one-year (T-1) and five years (T-5) preceding $T_{0}$. If data were not available for the appropriate age match, the closest data point that approximated an age-matched $\mathrm{T}_{0}$ that also allowed capturing blood pressure values at least one-year prior to this time

Table 1: Demographics, including gender, race, and mean age-of patients included in the analysis. Mean age-of patients was recorded as the age at which dementia was diagnosed. The control group of patients without dementia was age-matched with the dementia group.

\begin{tabular}{ccc}
\hline & Patient demographics & \\
\hline Parameters & Dementia & Without Dementia \\
\hline Mean age \pm SD & $81 \pm 7.3$ & $80.4 \pm 5.4$ \\
\hline Female & Gender, N & \\
\hline Male & 84 & 131 \\
\hline & 25 & 41 \\
\hline African American & Race, N & 41 \\
\hline Caucasian & 50 & 131 \\
\hline
\end{tabular}


point was utilized. If values were available for T-1 or T-5, but not both, patients were still included in the analysis but only for the available data points. By this method, the non-dementia patient means age was $-80.4 \pm 5$.4-yrs. All subjects were age-matched within the data range. Descriptive statistical analysis was performed in Excel. Graphs, Chi-Square analysis, and odds ratio calculations were generated with GraphPad Prism.

\section{RESULTS}

At the conclusion of data extraction, a total of 201 patients had data available for at least the 1-year period preceding the diagnosis of dementia. 80 of the original 281 patients did not have any prediagnosis data available and were not included in the analysis. Of the remaining 201, only 164 patients had available data for the full five-year pre-diagnosis period. Changes in blood pressure over each time period were calculated individually for each patient. Statistical analysis of blood pressure values revealed that the mean change in systolic blood pressure over the five years preceding dementia diagnosis was $-9.8 \pm 24.4 \mathrm{mmHg}$, while patients without a diagnosis of dementia showed a mean change of $0.35 \pm 30.0 \mathrm{mmHg}$, a statistically significant difference $(\mathrm{p}=0.001)$ (Figure 1 , Table 2$)$. The mean change of systolic blood pressure over the one-year preceding dementia diagnosis was $-8.1 \pm 21.6 \mathrm{mmHg}$, compared to a mean change of $-0.64 \pm 21.6 \mathrm{mmHg}$ for those without dementia $(\mathrm{p}=0.001)$.

Further analysis revealed that $67.2 \%$ of patients that developed dementia showed a decline in systolic blood pressure over the five years preceding diagnosis, while $46.6 \%$ of the group without dementia showed a decline over the same time period (Table 3 ). Additionally, $67.2 \%$ of patients with a dementia diagnosis showed a decrease in diastolic blood pressure over the previous five years, compared to $51.0 \%$ of those without dementia over the same period. $62.7 \%$ of those with dementia showed a decreasing systolic blood pressure over the one-year period preceding diagnosis, compared to $50.0 \%$ of the group without dementia. Finally, $52.2 \%$ of patients with dementia showed a decrease in diastolic blood pressure over year preceding diagnosis, compared to $50.0 \%$ of those without dementia. However, these changes in diastolic pressures were not found to be significant between the groups. Figure 1 displays the progressions of the mean systolic blood pressure for each study group over the five years preceding dementia diagnosis or comparable date for those without dementia. Odds ratio calculations revealed the odds of dementia patients to have had a declining systolic pressure over the five years preceding diagnosis to be 2.35 (CI $(95 \%)=1.21-4.45)$.

Table 2: Mean change in systolic and diastolic blood pressures for the "With Dementia" and "Without Dementia" groups from 5-years (or 1-year) prior to diagnosis. The control group of patients without dementia was age-matched with the dementia group.

\begin{tabular}{lcc}
\hline \multicolumn{3}{c}{ Blood pressure change preceding diagnosis } \\
Variables & $\begin{array}{c}\text { Systolic } \pm \text { SD } \\
(\mathbf{m m H g})\end{array}$ & $\begin{array}{c}\text { Diastolic } \pm \text { SD } \\
(\mathbf{m m H g})\end{array}$ \\
\hline \multicolumn{3}{c}{ 5-years prior to diagnosis } \\
\hline With Dementia & $-9.8 \pm 24.4$ & $-5.0 \pm 12.7$ \\
\hline Without Dementia & $0.35 \pm 30.0$ & $-0.31 \pm 16.7$ \\
\hline \multicolumn{3}{r}{ 1-year prior to diagnosis } \\
\hline With Dementia & $-8.13 \pm 21.6$ & $-3.1 \pm 10.3$ \\
Without Dementia & $-0.6 \pm 21.6$ & $-0.19 \pm 11.9$
\end{tabular}

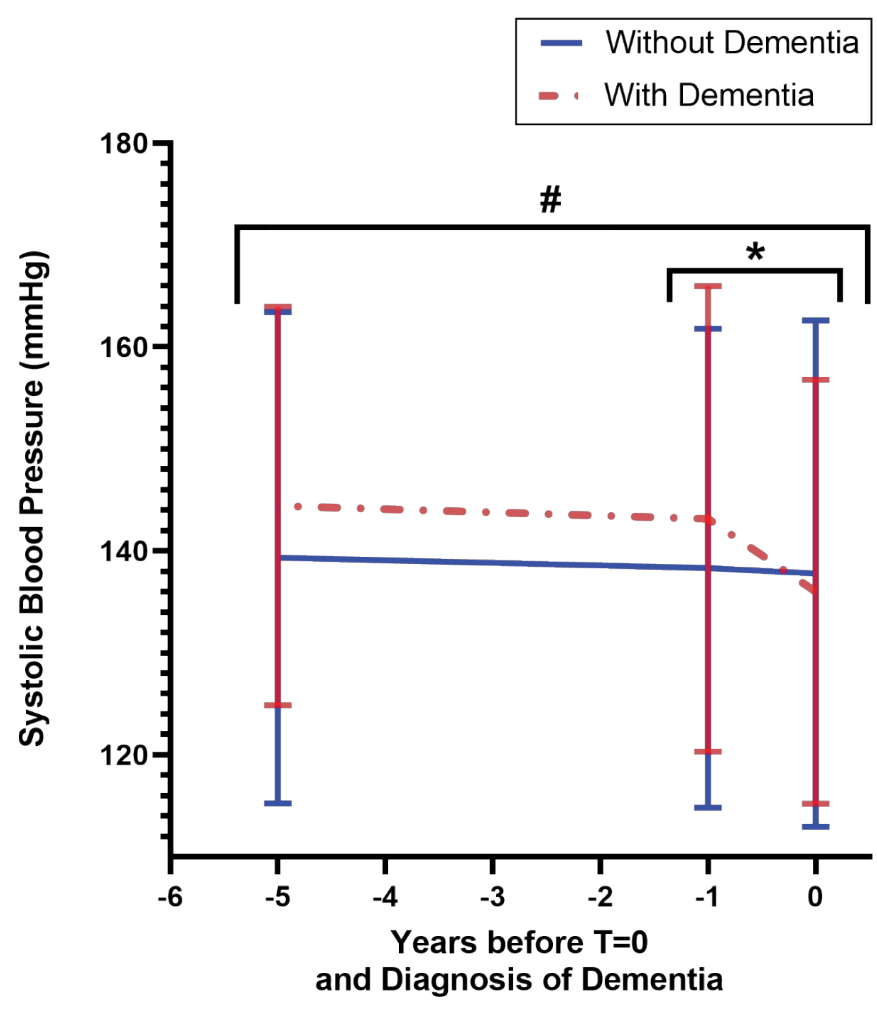

Figure 1: Trends in systolic blood pressure change over a five year period before the diagnosis of dementia. Patients with dementia had a sharp reduction in systolic blood pressure one year preceding the diagnosis of dementia vs. those without dementia, in whom the systolic blood pressure remained stable. \# $\mathrm{p}<0.001$ Difference in systolic blood pressure in patients with dementia vs. non-dementia over five years prior to diagnosis. ${ }^{*} \mathrm{p}<0.001$ Difference in systolic blood pressure in patients with dementia vs. non-dementia over one year prior to diagnosis. $\mathrm{T}=0$ is the time period during their clinical follow-up when patients were either diagnosed with dementia or did not have that diagnosis in their chart. The control group of patients without dementia were age-matched with the dementia group.

Table 3: Percent of patients in the "With dementia" or "Without dementia" group with decreasing systolic or diastolic blood pressures at 5-years or 1-year prior to diagnosis. The control group of patients without dementia was age-matched with the dementia group.

\begin{tabular}{ccc}
\hline \multicolumn{3}{c}{ Patients with decreasing blood pressures prior to diagnosis } \\
\hline Variables & Systolic (\%) & Diastolic (\%) \\
\hline \multicolumn{3}{c}{5 years prior to diagnosis } \\
\hline With Dementia & $67.2 \%$ & $67.2 \%$ \\
\hline Without Dementia & $46.6 \%$ & $51.0 \%$ \\
\hline \multicolumn{3}{c}{ 1 year prior to diagnosis } \\
\hline With Dementia & $62.7 \%$ & $50.0 \%$ \\
\hline Without Dementia & $52.2 \%$ & $50.0 \%$ \\
\hline
\end{tabular}

\section{Differences between African Americans and Caucasians}

An additional analysis was performed to compare the rates of dementia amongst racial groups, specifically African Americans and Caucasians. Descriptive statistics revealed that $55.0 \%$ (50/91) of African Americans in this data set developed dementia. In comparison only 31.0\% (59/131) of Caucasians developed dementia (Figure 2). Odds ratio calculation for African American development of dementia was calculated to be 2.71 (CI $(95 \%)=1.625-4.487)$.

Of the African American patients, 59.4\% of those who developed 
dementia had a decrease in systolic and diastolic pressure over the five-year pre-diagnosis period compared to $41.4 \%$ those in the group without dementia. Of the Caucasian patients, $75.9 \%$ of those with dementia had a decrease in systolic and diastolic pressure over the five-year period compared to $48.7 \%$ of patients without dementia. In comparison, $68.6 \%$ of African American patients with dementia had a decrease in systolic and diastolic pressures over the one-year period compared to $47.1 \%$ of those without dementia, while only $54.5 \%$ of Caucasian patients with dementia had a decrease in systolic and diastolic pressures over the one-year period compared to $51.0 \%$ of Caucasian patients without dementia.

Further analysis by racial subgroup showed a mean reduction in systolic blood pressure over five years of $-6.8 \pm 25.8 \mathrm{mmHg}$ and over the one-year period of $-10.4 \pm 21.2 \mathrm{mmHg}$ for African American patients preceding the diagnosis of dementia. In comparison, the mean reduction for Caucasians was $-13.2 \pm 22.7 \mathrm{mmHg}$ over five years and $-5.6 \pm 22.1$ over the one-year period. The changes in the mean systolic blood pressure for each subgroup are displayed in Figure 3. Comparison of African American versus Caucasian mean change revealed a statistically significant difference over the five-year period $(p<0.001)$, but not the one-year period $(p=0.26)$. Changes in the mean diastolic blood pressure were not significant between the subgroups.

\section{DISCUSSION}

This study analyzing the blood pressure trends preceding a diagnosis of dementia in heart failure patients found that those patients that developed dementia were more likely to have experienced a decrease in both systolic and diastolic blood pressure over the five-year period preceding dementia compared to those

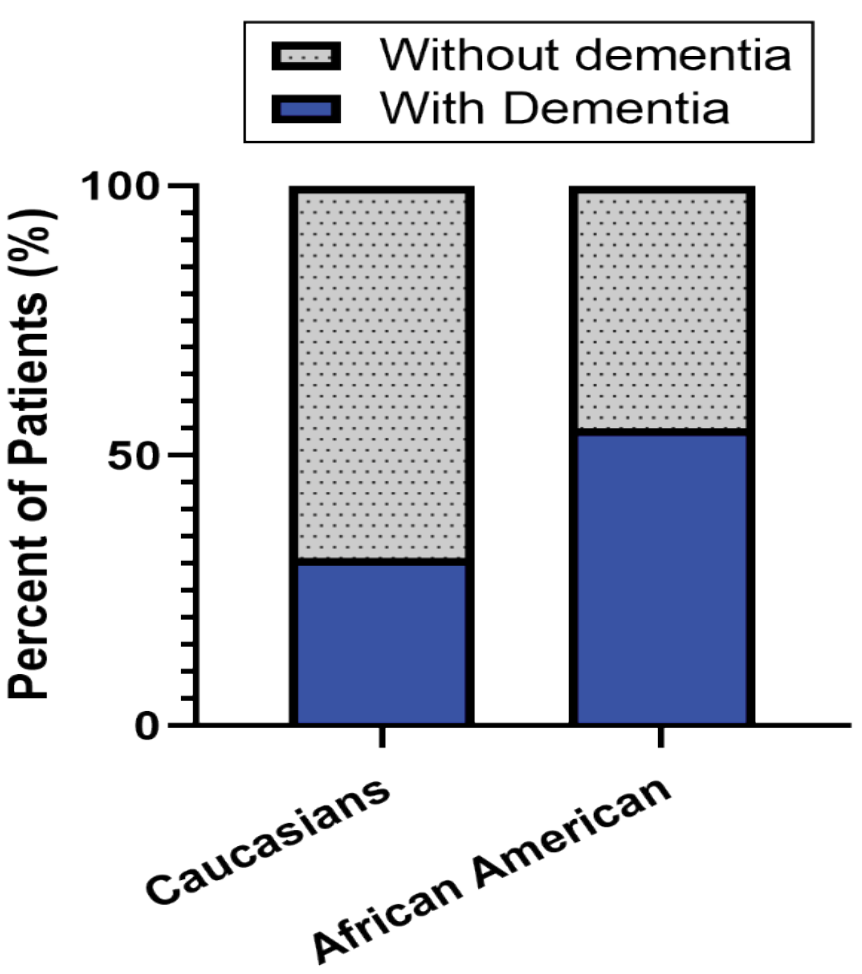

Figure 2: Proportion of patients diagnosed with dementia vs. those who had no dementia diagnosis in African Americans and Caucasians. A higher percentage of African Americans had a diagnosis of dementia listed in their medical record compared to Caucasians (O.R. $=2.71)$.

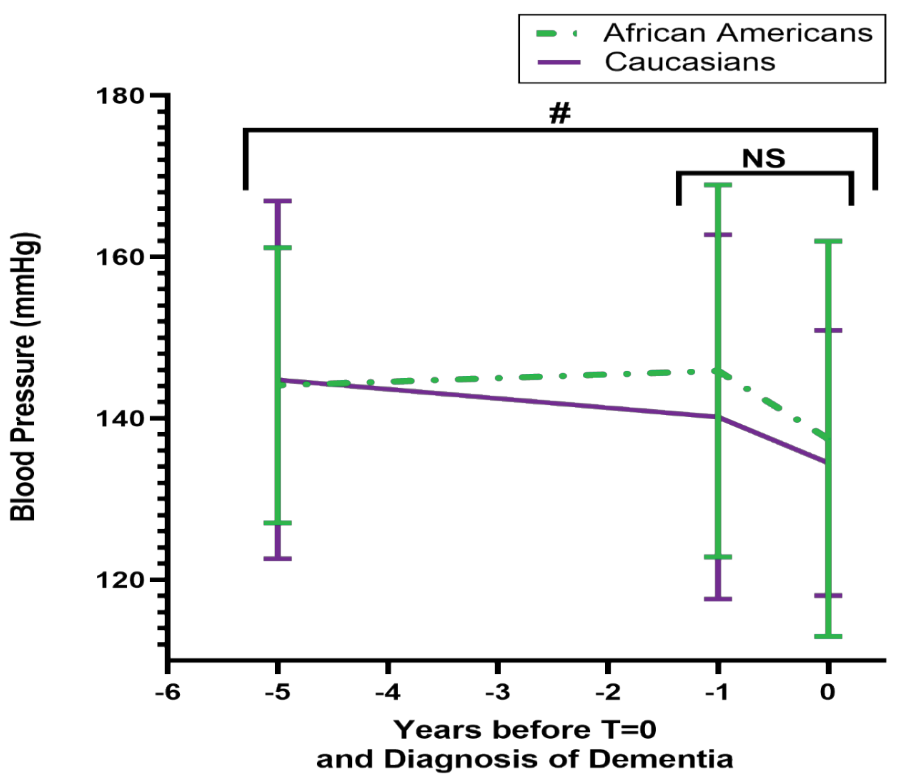

Figure 3: Five year trends in systolic blood pressure changes before a dementia diagnosis in African Americans vs. Caucasians. Caucasians experienced a greater overall reduction in systolic blood pressures over the five years preceding the diagnosis of dementia compared to African Americans $(\#=p<0.001)$. However, African Americans had a greater reduction in systolic pressures during the last year before dementia diagnosis compared to Caucasians (NS = non-significant). $\mathrm{T}=0$ is the time period during their clinical follow-up when patients were either diagnosed with dementia or did not have that diagnosis in their chart. The control group of patients without dementia were age-matched with the dementia group.

without dementia of a similar age $(\mathrm{OR}=2.35)$. Lip et al. and Schmid et al. both describe that in heart failure patients, the relationship between all-cause mortality and blood pressure demonstrated a J-curve, meaning both those with exceptionally low and high blood pressure were at greater risk of mortality $[8,9]$. An additional study showed that higher systolic blood pressure was a favorable prognostic factor in congestive heart failure [14]. It is possible that a similar relationship exists for the onset of dementia. In the current retrospective study, a decline in cognitive health was associated with a decreasing systolic blood pressure. Whether this decrease in systolic pressure is a marker for severe disease, comorbid illness, or an alternative hypothesis is difficult to say with the available data, but an odds ratio of 2.35 is not insignificant and requires further investigation.

Additionally, it was determined that the mean changes in both five-year and one-year systolic blood pressure for patients with a diagnosis of dementia and those without dementia were statistically significant at the $99 \%$ confidence interval $(p=0.001)$. This demonstrates that not only were patients with dementia more likely to have a decreasing systolic blood pressure, but they were also more likely to have a greater magnitude of decline compared to those without dementia. Furthermore, analysis also demonstrated that the magnitude of blood pressure changes for dementia patients revealed a more rapid decline over the one-year period compared to the five-year period. This is illustrated by the change of systolic blood pressure, as the five-year difference was $-9.83 \pm$ $24.4 \mathrm{mmHg}$ while the change over the one-year period was $-8.1 \pm$ $21.6 \mathrm{mmHg}$. This indicates that a more than $80 \%$ of mean change in systolic blood pressure for these patients occurred in the one- 
year preceding dementia, a phenomenon displayed well in Figure 1. The reason for the decline in systolic blood pressure cannot be determined clearly from our retrospective data. However, based on other foundational research regarding the hypoperfusion of the brain and the onset of dementia, it is plausible that such a decrease in systolic blood pressure could contribute to the pathogenesis of dementia in certain patient populations [2]. Additionally, this observation that rapidly declining systolic blood pressure during later-life may precede dementia is in concordance with findings of McGrath et al [4]. Because heart failure is a syndrome of chronic hypoperfusion and cyclical compensatory changes, it is likely that this population of patients would be at greater risk of the effects of declining blood pressure.

Alternatively, because we now know that the dementia process often begins more than 10 to 20 years earlier, it may be that the reduction in systolic blood pressure one-year before the clinical diagnosis is made may actually herald a progression in the stage of the cognitive impairment to a more advanced level $[15,16]$.

An additional finding worthy of discussion is the disparity of dementia rates among African Americans and Caucasians in this data set. It was found that those who developed dementia were significantly more likely to be African American than Caucasian $(\mathrm{OR}=2.71)$. These groups were not determined to be statistically different in mean age or BMI, although it was identified that African Americans had significantly higher rates of hyperlipidemia (78.0\% vs. $24.7 \%$ ) and hypertension $(94.5 \%$ vs. $83.7 \%)$, two known risk factors for dementia, as compared to Caucasians. It was determined that of those who developed dementia, Caucasians experienced a significantly greater five-year magnitude of change in mean systolic blood pressure compared to their African American counter parts $(-13.2 \pm 22.7$ vs. $-6.8 \pm 25.8$; $p<0.001)$, while the oneyear magnitudes where not significantly different $(-10.4 \pm 21.2$ vs. $-5.6 \pm 22.1 ; \mathrm{p}=0.26)$. Inequalities of dementia incidence have been demonstrated before as Mayeda et al. have previously shown that the incidence of dementia is higher in African Americans at 26.6/1000 compared to $19.3 / 1000$ in Caucasians [17]. The significance of social determinants of health, such as race, are often difficult to integrate into our understanding of a disease process, but it is crucial that we be aware of disparities in rates of disease and the influence that distal risk factors have on our patient's health. In this case, African Americans had a significantly higher rate of dementia compared to their Caucasian counterparts. It is possible that this is due to greater rates of co-morbidities such as hypertension and hyperlipidemia. Even so, health care providers should be aware of this trend and work to mitigate these and other risk factors in order to diminish such disparity.

Healthcare providers are generally good at monitoring increases in blood pressure, but they may often fail to note downward trends in systolic blood pressure, or they may consider the decrease in blood pressure benign. However our data from this retrospective study shows it is very important for providers to monitor any downward trends in systolic pressures because it can precede the development of dementia even over a short duration of five years. Patients and caregivers also need to be aware of the risks associated with a reduction in blood pressure. Sometimes the reason for low blood pressure may include an illness leading to weight loss, an inappropriate regimen of blood pressure medications, polypharmacy, or cardiac conditions such as heart attack or exacerbation of heart failure. From this retrospective study, we did not have sufficient information to look at all of the potential causes of low blood pressure or cognitive decline for all of these patients. However, regardless of the cause of reduced systolic blood pressure, healthcare providers and caregivers need to be monitoring these patients for subtle changes in cognition if there is an unusual drop in systolic blood pressure due to medications or comorbid conditions.

\section{Limitations and strengths of the study}

The limitations of this study included its retrospective nature and the small sample size from a largely rural area in the United States. These limitations dictated what data and during what time intervals data were accessible from the patient records as well as restricting how representative the sample population was of the entire country.

Nonetheless, this study demonstrated a few strengths. These strengths included a more even diversity than the national average, as African Americans made up $45 \%$ of our study cohort as compared to $12 \%$ on a national level. Additionally, the primary clinic from which these retrospective data derived is one of the largest geriatric out-patient clinics in the country. Geriatricians may screen more often for dementia compared to other primary care providers, possibly allowing our data to be a more diverse and thorough representation of the local community.

\section{CONCLUSIONS AND RECOMMENDATIONS}

Dementia and heart failure are both complex diseases with a multifactorial pathogenesis. They are both diagnoses that exert a significant burden on society and warrant the attention of physicians and scientists. The phenomena highlighted in this report are important to propel the discussion of a multisystem approach to dementia and the influence of low blood pressure in heart failure. It is possible that subjects in the dementia group had some cognitive impairment for years before their diagnosis. However, the decline in systolic blood pressure could have contributed to worsening of their cognition. We hope that providers will have greater awareness of the potential dangers of lowering blood pressure too aggressively in elderly patients and be cognizant of the fact that African Americans may be at greater risk of developing dementia compared to their Caucasian counterparts.

\section{ACKNOWLEDGEMENTS}

We thank Codi English for help with manuscript preparation. This study was partly supported by PHS\# AG028718.

\section{REFERENCES}

1. Matthews KA, Xu W, Gaglioti AH, Holt JB, Croft JB, Mack D, et al. Racial and ethnic estimates of alzheimer's disease and related dementias in the United States (2015-2060) in adults aged $\geq 65$ years. Alzheimers Dement. 2019;15(1):7-24.

2. Wolters FJ, Zonneveld HI, Hofman A, Lugt VA, Koudstaal PJ, Vernooij MW, et al. Cerebral perfusion and the risk of dementia: Apopulation-based study. Circulation. 2017;36(8):719-728.

3. Gottesman RF, Albert MS, Alonso A, Coker LH, Coresh J, Davis $\mathrm{SM}$, et al. Associations between midlife vascular risk factors and 25 year incident dementia in the Atherosclerosis Risk in Communities (ARIC) cohort. JAMA neurology. 2017;74(10):1246-1254. 
4. McGrath ER, Beiser AS, DeCarli C, Plourde KL, Vasan RS, Greenberg $\mathrm{SM}$, et al. Blood pressure from midlto late life and risk of incident dementia. Neurology. 2017;89(24):2447-2454.

5. Oishi E, Ohara T, Sakata S, Fukuhara M, Hata J, Yoshida D, et al. Day-to-day blood pressure variability and risk of dementia in a general Japanese elderly population: The hisayama Study. Circulation. 2017;136(6):516-525.

6. Schaare HL, Masouleh SK, Beyer F, Kumral D, Uhlig M, Reinelt JD, et al. Association of peripheral blood pressure with gray matter volume in 19-to 40-year-old adults. Neurology. 2019;92(8):758-773.

7. Benjamin EJ, Virani SS, Callaway CW, Chamberlain AM, Chang AR, Cheng S, et al. Heart disease and stroke statistics-2018 update: A report from the American Heart Association. Circulation. 2018;137(12):467-492.

8. Lip GY, Skjoth F, Overvad K, Rasmussen LH, Larsen TB. Blood pressure and prognosis in patients with incident heart failure: The Diet, Cancer and Health (DCH) cohort study. Clin Res Cardiol. 2015; 104(12):1088-1096.

9. Schmid FA, Schlager O, Keller P, Seifert B, Huang R, Frohlich GM, et al. Prognostic value of long-term blood pressure changes in patients with chronic heart failure. Eur J Heart fail. 2017;19(7):837-842.

10. Adelborg K, Horvath $\triangle$ Puho E, Ording A, Pedersen L, Sorensen HT, Henderson VW. Heart failure and risk of dementia: A danish nationwide population】based cohort study. Eur J Heart Fail. 2017;19(2):253-260.

11. Rusanen M, Kivipelto M, Levalahti E, Laatikainen T, Tuomilehto J, Soininen $\mathrm{H}$, et al. Heart diseases and long-term risk of dementia and alzheimer's disease: A population-based CAIDE study. J Alzheimers Dis. 2014;42(1):183-191.

12. Qiu C, Winblad B, Marengoni A, Klarin I, Fastbom J, Fratiglioni L. Heart failure and risk of dementia and Alzheimer disease: A population-based cohort study. Arch Intern Med. 2006;166(9):1003. 1008 .

13. Jefferson AL, Beiser AS, Himali JJ, Seshadri S, O’Donnell CJ, Manning WJ, et al. Low cardiac index is associated with incident dementia and alzheimer disease: The Framingham Heart Study. Circulation. 2015;131(15):1333-1339.

14. Raphael CE, Whinnett ZI, Davies JE, Fontana M, Ferenczi EA, Manisty $\mathrm{CH}$, et al. Quantifying the paradoxical effect of higher systolic blood pressure on mortality in chronic heart failure. Heart. 2009: 95(1):56-62.

15. Abell JG, Kivimaki M, Dugravot A, Tabak AG, Fayosse A, Shipley M, et al. Association between systolic blood pressure and dementia in the whitehall II cohort study: Role of age, duration, and threshold used to define hypertension. Eur Heart J. 2018;39(33):3119-3125.

16. Samieri C, Perier MC, Gaye B, Proust-Lima C, Helmer C, Dartigues JF, et al. Association of cardiovascular health level in older age with cognitive decline and incident dementia. Jama. 2018;320(7): 657-664.

17. Mayeda ER, Glymour MM, Quesenberry CP, Whitmer RA. Inequalities in dementia incidence between six racial and ethnic groups over 14 years. Alzheimers Dement. 2016;12(3):216-224. 